Bull. Mater. Sci., Vol. 35, No. 1, February 2012, pp. 13-18. (c) Indian Academy of Sciences.

\title{
Effects of nitrogen annealing on surface structure, silicide formation and magnetic properties of ultrathin films of $\mathrm{Co}$ on $\mathrm{Si}(100)$
}

\author{
GANESH K RAJAN, SHIVARAMAN RAMASWAMY*, C GOPALAKRISHNAN \\ and D JOHN THIRUVADIGAL ${ }^{\dagger}$ \\ Nanotechnology Research Centre, ${ }^{\dagger}$ Department of Physics, SRM University, Kancheepuram District 603 203, India
}

MS received 19 August 2010; revised 16 September 2010

\begin{abstract}
Effects of nitrogen annealing on structural and magnetic properties of $\mathrm{Co} / \mathrm{Si}(100)$ up to $700^{\circ} \mathrm{C}$ has been studied in this paper. Ultrathin Co films having a constant thickness of $50 \AA$ were grown on Si (100) substrates using electron-beam evaporation under very high vacuum conditions at room temperature. Subsequently, the samples were annealed at temperatures ranging from $100-700^{\circ} \mathrm{C}$ in a nitrogen environment at atmospheric pressure. Sample quality and surface morphology were examined using atomic force microscopy. Silicide formation and the resultant variation in crystallographic arrangement were studied using X-ray diffractometer. The magnetization measurements done using a vibrating sample magnetometer indicate a decrease in coercivity and retentivity values with increase in annealing temperature. Resistivity of the samples measured using a four-point probe set up shows a decrease in resistivity with increase in annealing temperature. Formation of various silicide phases at different annealing temperatures and the resultant variation in the magnetic susceptibility has been thoroughly studied and quantified in this work.
\end{abstract}

Keywords. Cobalt; silicides; nitrogen annealing; AFM; XRD.

\section{Introduction}

As the size of integrated circuits decreases every year, metallization of the circuits becomes an important factor to be considered. This has fuelled the need for developing materials with good mechanical properties, low resistivity and high temperature and chemical stability. In the past few decades, cobalt silicides has been extensively used for the metallization of very large integrated circuits such as ohmic contacts, interconnects, Schottky barriers and electrodes. The advantages of $\mathrm{CoSi}_{2}$ in forming contacts to silicon in CMOS devices have been vastly investigated in the past few decades. Compared to nickel, silicide formation of Co is less sensitive to the native oxide formation at the interface (Lavoie et al 2002; Froment et al 2003). Also, the ferromagnetic nature of cobalt makes it a suitable candidate for the magnetic recording industry. It is well known that in the case of metal silicides the post deposition processing plays a major role in tuning the electrical and magnetic properties of the magnetic nanostructures.

It is known that ferromagnetic cobalt layers deposited epitaxially on Si (100) substrate exhibit in plane and perpendicular magnetism over a broad thickness range which leads to complex spin reorientation mechanism (Tsay et al 2002; Chang et al 2007; Wernsdorfer et al 2009). Further it is

\footnotetext{
*Author for correspondence (shivi.masti@gmail.com)
}

known that most metals are not in thermodynamic equilibrium with silicon. Hence, spontaneous and subsequent intermixing occurs in metals films on $\mathrm{Si}$ resulting in the formation of various intermediate phases (Lau et al 1978; Joensson et al 2006). The characteristics at the interface are dictated by uniformity of interdiffusion parallel to interface, interdiffusion thickness and structural properties at the interface. Even though a number of fundamental studies based on these phenomena have been carried out in the past few decades, for small thicknesses $(<50 \mathrm{~nm})$, the effects of annealing in a nitrogenous environment are neither documented nor are they completely understood. It is well understood that the effects of annealing of $\mathrm{Co} / \mathrm{Si}$ film in various ambients would be different.

In the present work, we have done a detailed quantitative investigation of the variation in magnetic, structural and electrical properties with respect to the annealing temperature in ultra thin Co films annealing in nitrogen ambient at atmospheric pressures. The silicide formation at different temperatures is also discussed in detail on the basis of XRD data.

\section{Experimental}

Silicon wafers of $10 \times 10 \times 0.5 \mathrm{~mm}$ were thoroughly cleaned using the standard RCA cleaning procedure. According to this procedure, the silicon substrates $(1 \times 1 \mathrm{~cm})$ were thoroughly cleaned and degassed using high temperature ultrasonication in acetone. 
The deposition was carried out in a commercially procured physical vapour deposition system under a pressure of $5 \times 10^{-6} \mathrm{mBar}$. A Varian V500 turbo molecular pump was used to establish the very high vacuum. Prior to deposition, the samples were etched using high energy argon ions and annealed to obtain a smooth and impurity free surface. The deposition was done within a very short time after argon ion etching in order to reduce the oxide contamination. A $3 \mathrm{kV}$ sweeping mode electron beam gun was used to collimate the high energy electrons onto the high purity $(99.99 \%$ pure) bulk cobalt which was held in water cooled copper crucibles. All the samples were coated with $50 \AA$ of cobalt at a rate of $0 \cdot 1 \AA /$ s. The deposition rate was controlled using an Inficon controller and a quartz crystal based digital thickness controller. The samples were then annealed in nitrogen ambient at atmospheric pressure at temperatures ranging between 100 and $700^{\circ} \mathrm{C}$ for a duration of 90 min with a progressive increase of $100^{\circ} \mathrm{C}$ per sample.

Morphology of the sample was studied using Agilent Technologies atomic force microscope (AFM). All images were taken in contact mode using silicon cantilevers with a force constant of $0.02-0.77 \mathrm{~N} / \mathrm{m}$, tip height, $10-15 \mu \mathrm{m}$. The root mean square (RMS) roughnesses of the samples were calculated from AFM images. The phase purity, silicide formation and crystallographic orientation of the samples were studied using PANalytical's Xpert Pro X-ray diffractometer (XRD) in grazing incidence (GI) geometry. The instrument uses a $\mathrm{CuK} \alpha$ radiation, $(\lambda=)$ and the measurements were recorded in the $2 \theta$ range of $10-60^{\circ}$. Resistivity of the film was measured using the four-point probe (FPP) sheet resistance measurement set up. For electrical measurements of the sample, silver paste was applied at the corners of the wafer to provide electrical contacts. Magnetic properties of the samples were analysed using Lake Shore's vibrating sample magnetometer (VSM) and magnetic flux density vs magnetizing force $(\mathrm{B}-\mathrm{H})$ loop plotter.

\section{Results and discussion}

The various cobalt silicide phases $\left(\mathrm{Co}_{x} \mathrm{Si}_{y}\right)$ present in a series of pristine and annealed samples were characterized using XRD in GI mode. The results are consolidated in figure 1. The X-ray diffractogram of the as deposited specimen gives the Bragg's peak at $45.9^{\circ}$ and $53^{\circ}$ corresponding to the (211) peaks of $\mathrm{Co}_{2} \mathrm{Si}$ and the (002) peak of hexagonal $\mathrm{Co}$, respectively. The formation of $\mathrm{Co}_{2} \mathrm{Si}$ in the as-deposited sample indicates that cobalt atoms were available in plenty for the silicon atoms to form the cobalt rich silicide. The deposition of $50 \AA$ typically results in several monolayers of cobalt deposition over the silicon substrate. This and the fact that e-beam evaporation was used (which results in higher mobility of atoms on the surface due to high temperature) make the formation of cobalt rich silicide the most preferred silicide in the absence of any post-treatments. Also, the peak corresponding to the substrate is visible at $55^{\circ}$. In samples annealed up to $100,200,300$ and $400^{\circ} \mathrm{C}$, the intensity of the
$\mathrm{Co}(002)$ and $\mathrm{Co}_{2} \mathrm{Si}$ (211) peaks were found to decrease progressively. This is indicative of diffusion of cobalt atoms into the silicon matrix, which is characteristic of the annealing process. In the samples which were annealed up to $500^{\circ} \mathrm{C}$, along with the $\mathrm{Co}$ and $\mathrm{Co}_{2} \mathrm{Si}$ peaks, the (210) and (211) phases of $\mathrm{CoSi}$ were also visible at $53.871^{\circ}$ and $59.386^{\circ}$, respectively. Further, there was a distinct reduction in the intensities of $\mathrm{Co}$ and $\mathrm{Co}_{2} \mathrm{Si}$ peaks. This can be attributed to the fact that, upon annealing, there is an increased diffusivity and due to the resultant shortage in the cobalt atoms, monosilicides are formed instead of di-metal silicides. At annealing temperatures higher than $600^{\circ} \mathrm{C}$, the diffraction peaks of (220) phase of $\mathrm{CoSi}_{2}$ was significantly visible at a $2 \theta$ value of $57^{\circ}$ along with CoSi peaks.

The reduced intensity of CoSi peaks denotes further diffusion of the cobalt atoms into the silicon matrix at high temperatures. Also, at this point the peak corresponding to pure cobalt (002) was found to be completely absent. This is indicative of the fact that all the cobalt atoms were used up for the formation of silicides. Further, the absence of cobalt nitride and silicon nitride peaks in XRD is indicative of the absence of the same in the sample. This implies that nitrogen adsorption, if any, did not result in chemical or stoichiometric changes or changes in the crystalline nature of the sample.

Figure $2(\mathrm{a}-\mathrm{h})$ shows AFM images of the as deposited and annealed samples. It can be seen that the as-deposited sample follows the standard Volmer Weber growth mode. Initially, individual islands of cobalt are formed on the silicon substrate. This is typical of a nucleation driven growth mode. Line profile analysis done on the AFM images show that the average size of the structures is around $40 \mathrm{~nm}$. Compared to the as-deposited sample, samples annealed at $100^{\circ} \mathrm{C}$ showed a reduction in the average density of distribution of the particles. This was observed to decrease further in samples annealed at 200,300, 400 and $500^{\circ} \mathrm{C}$ (figures $2 \mathrm{~b}, \mathrm{c}, \mathrm{d}$ and e), respectively. Also, it was noticed that the film became more continuous for samples annealed above $300^{\circ} \mathrm{C}$. In correlation with XRD data, reduction in the density of distribution of the cobalt islands can be attributed to the diffusion of metallic cobalt into the silicon matrix and the subsequent formation of silicides upon annealing. Another change that can be noted in the structures is the increase in the average size of the nanostructures upon annealing. This can be attributed to the fact that upon annealing, the mobility of the atoms on the surface of the substrate increases due to thermal energy. These mobile atoms prefer to be in the lowest energy state thereby nucleating and forming larger structures by agglomerating around the nucleation sites. Further, the annealing process tends to support the formation of cobalt silicides rather than elemental cobalt nanostructures. It can be clearly seen from the AFM images that in case of the samples annealed above $500^{\circ} \mathrm{C}$, both the spatial distribution of particles and the particle size distribution were quite random. This is probably due to the prolonged process of the nucleation and supported growth. We can see that, in the samples annealed above $500^{\circ} \mathrm{C}$ instead of existing 


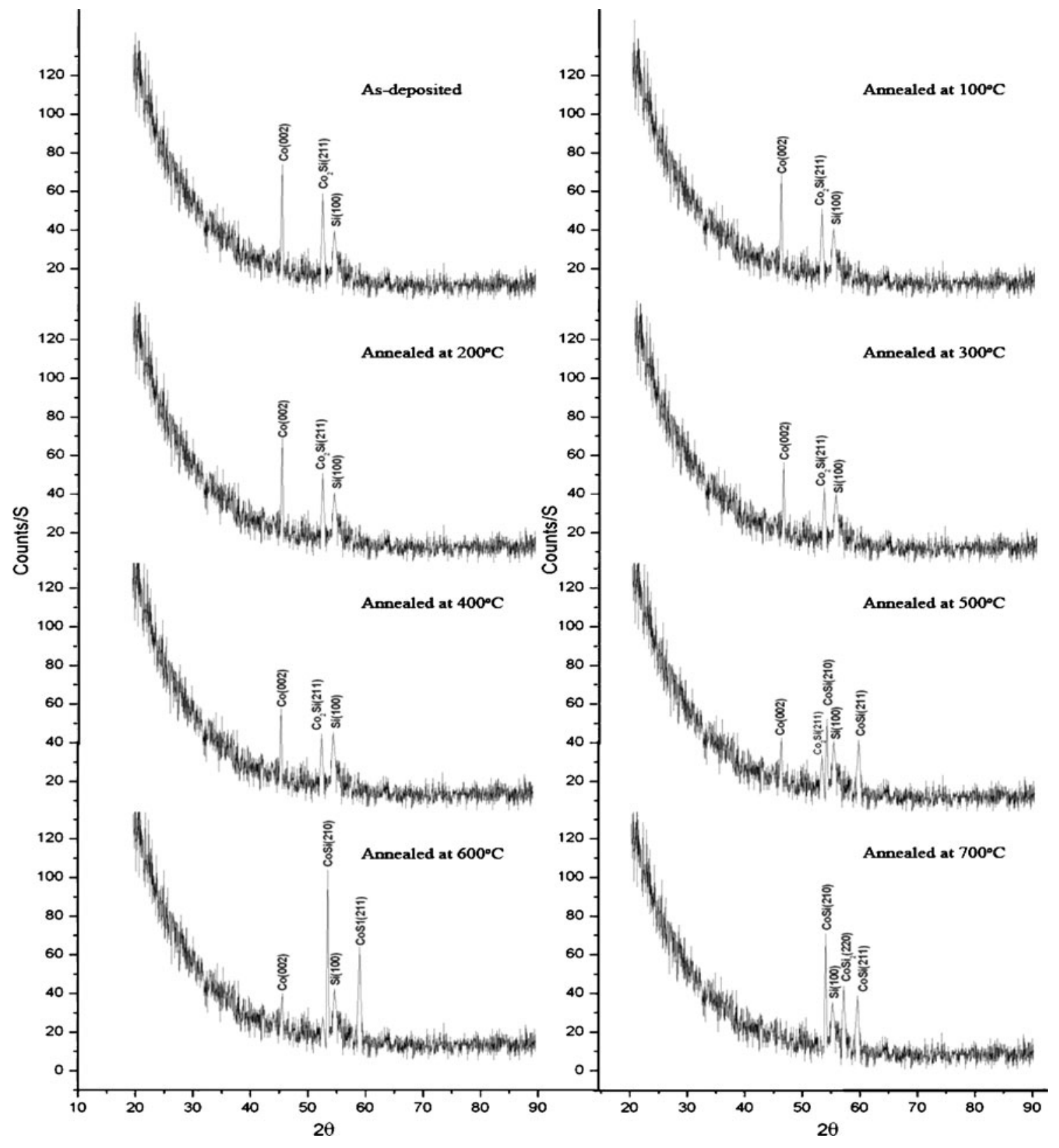

Figure 1. XRD data of as deposited and annealed samples.

as distinct particles, the structures were in the form of two dimensional sheet-like structures. The sheet-like appearance of the sample at high temperatures can be attributed to the stress experienced by thin film as a result of the nitrogen annealing process. Roughness histograms taken for the AFM images (data not shown) indicate that at elevated temperatures $\left(300-500^{\circ} \mathrm{C}\right)$, there is a decrease in the roughness of the film probably as a result of the surface atom mobility and the formation of nanosheets. In contrast, samples annealed at yet higher temperatures $\left(600^{\circ} \mathrm{C}\right.$ and $\left.700^{\circ} \mathrm{C}\right)$ become rougher, due to the formation of larger isolated grains (Agarwal et al 2007a, b). This is in good agreement with the AFM images shown below.
Resistivity measurements done on the sample (figure 3 ) ascertains the fact that the grains were distinct in the as-deposited sample whereas they coalesce into bigger nanostructures such as two-dimensional nanosheets upon annealing. The as-deposited film shows a high resistivity which is seen to decrease as the annealing temperature increases. Also it is well known that, another major factor in the resistivity of thin films is the adsorbed gases, if any. It is obvious that prolonged annealing would lead to the adsorption of nickel onto the cobalt film. This would typically lead to high resistivity as in the as-deposited films (750 micro ohms $/ \mathrm{cm}$ ). The resistivity can be attributed to this adsorption which would severely reduce the carrier mobility. As 


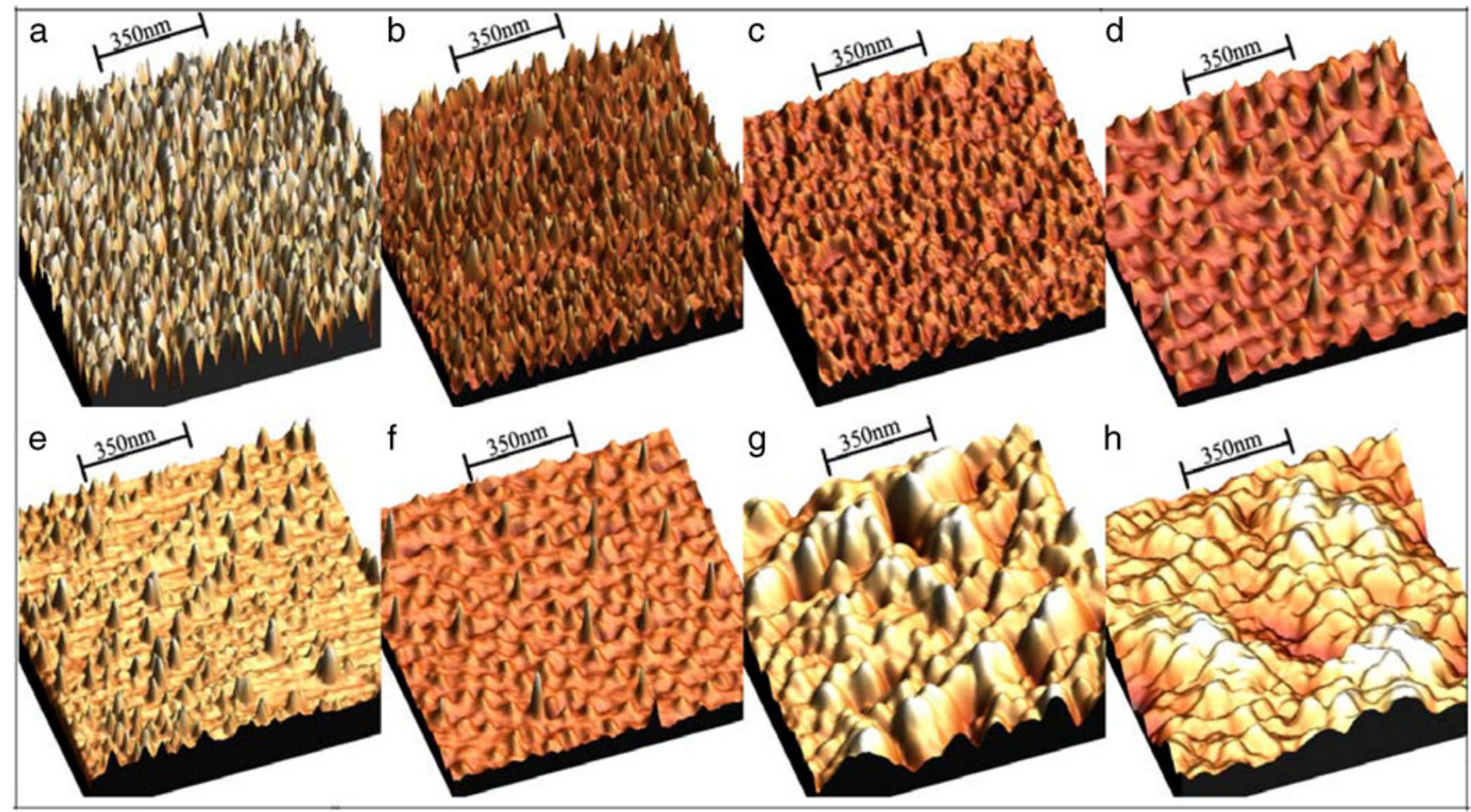

Figure 2. 3-D AFM images of a. as-deposited samples and samples annealed at $100-700^{\circ} \mathrm{C}$ with a progressive increase of $100^{\circ} \mathrm{C}$ per sample (b-h).

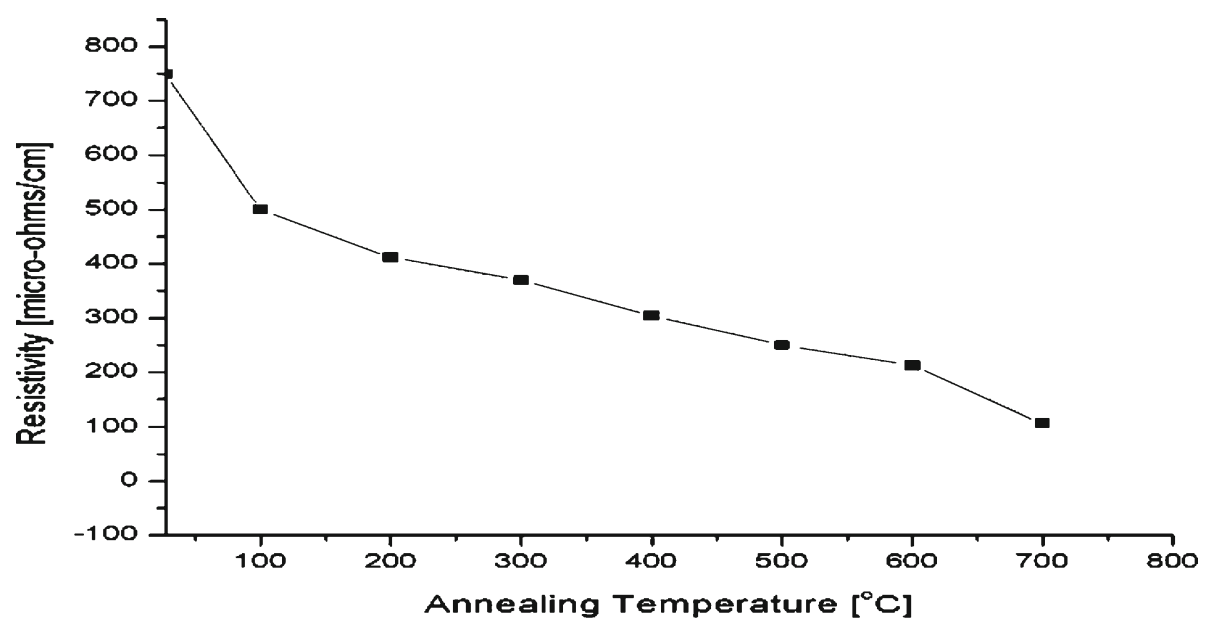

Figure 3. Resistivity of $\mathrm{Co} / \mathrm{Si}$ plotted as a function of annealing temperature.

the temperature of annealing increases this also allows for the nitrogen to diffuse out in a process similar to outgassing which would in turn result in a decrease in the resistivity of the film. Further, as the temperature increases $\left(100,200,300,400\right.$ and $\left.500^{\circ} \mathrm{C}\right)$, the grains grow bigger in size, forming continuous films which would have significantly lesser resistance than distinct two dimensional islands as seen in the as-deposited films. Also, the defect density in the as deposited films would be much higher than the annealed ones, which could also contribute to a higher resistivity.

The magnetic properties of the samples were characterized by ex-situ VSM at room temperature with the measurements taken normal to the plane of the samples. The VSM was calibrated using a nickel sphere and gadolinium oxide powder, and the resolution of VSM was about $1 \times 10^{-7}$ emu. Measurements were performed by applying a magnetic field of 3000 Oe in plane to the substrate. The analysis of data 


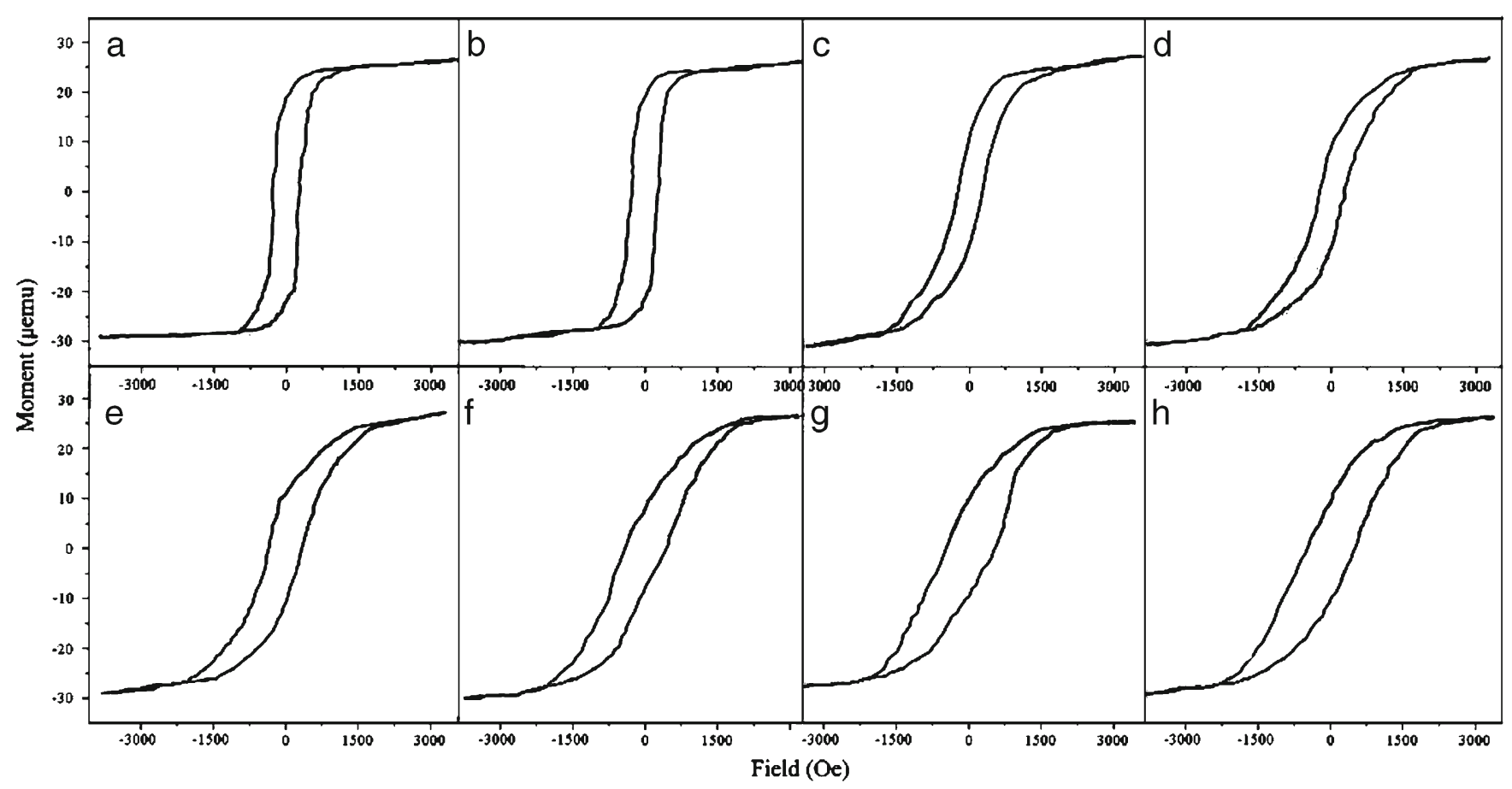

Figure 4. Magnetization loops of a. as deposited and annealed (b-h) samples.

was done after subtracting the substrate and holder offset as described by previous works (Diaz-Castanon et al 2008; Ramaswamy et al 2010a, b). From the recorded hysteresis loops (figure $4(a-h)$ ), it has been observed clearly that all the samples showed typical ferromagnetism as expected from the cobalt samples.

Further, it can be seen that the hysteresis loop of asdeposited and samples annealed at lower temperatures are more rectangular in shape as compared to the samples annealed at higher temperatures. That is, the squareness of the hysteresis loop decreases with increase in annealing temperature. This is indicative of the fact that there exists an easy axis of magnetization normal to the plane of the sample in the as-deposited samples. This can be typically correlated as being caused due to the predominant effect of shape anisotropy as is the case in one dimensional islands (Ramaswamy et al 2010c, d; Rao et al 2010). Further, the decrease in the squareness can be attributed to the formation of larger magnetic clusters as can be seen in AFM images. This in turn leads to a rotation of the easy axis of magnetization as the cobalt rich silicides are replaced by silicon rich silicides $\left(\mathrm{Co}_{2} \mathrm{Si}\right.$ by $\mathrm{CoSi}$ and $\mathrm{CoSi}_{2}$ ). Such changes in the crystallographic phases and the orientation of cobalt would typically be accompanied by changes in the magnetocrystalline anisotropy. The competing influences of the shape anisotropy of the two dimensional sheets and the magnetocrystalline anisotropy originating from the sample can be attributed to the reduction of the squareness of the hysteresis loops with increase in annealing temperature. Further research is underway to better under- stand this phenomenon. It can also be seen that the coercivity increases and the retentivity decreases with increase in annealing temperature. These results are consistent with our expectation of thin films subjected to the annealing process.

\section{Conclusions}

As deposited and annealed samples of $\mathrm{Co} / \mathrm{Si}$ were studied using grazing-incidence X-ray diffraction (GIXRD), vibrating sample magnetometer (VSM), atomic force microscopy (AFM) and four-point probe resistivity measurement system (FPP). Samples were annealed at various temperatures in nitrogen environment at atmospheric pressures to study the diffusivity of cobalt into the substrate layer and the subsequent silicide formation. XRD results revealed a metal deficient disilicide $\left(\mathrm{CoSi}_{2}\right)$ formation along with a minor phase of $\mathrm{CoSi}$ at the interface at annealing temperatures at and above $600^{\circ} \mathrm{C}$. Further, a significant increase in the conductivity has been observed for samples annealed at higher temperatures which was based on the morphological changes in the sample and the effect of nitrogen annealing on the samples. AFM images confirmed increase in the nanocrystalline grain size and the conclusions drawn by other techniques. The study of the magnetic properties indicated an increase in the coercivity and rotation of the easy axis of magnetization as a result of combined effect of shape anisotropy and magnetocrystalline anisotropy caused due to the 
formation of larger grains as a result of high temperature annealing.

\section{Acknowledgements}

The authors thank SRM University, Chennai, for financing this project and also for providing infrastructure facilities for carrying out these experiments.

\section{References}

Agarwal S, Ganesan V, Tyagi A K and Jain I P 2007a Bull. Mater. Sci. 29647

Agarwal S, Jain A, Karar N, Chakraborthy B R, Gupta A and Jain I P 2007b J. Scanning Probe Microsc. 36

Chang H W, Tsay J S, Hung Y C, Yuan F T, Chan W Y, Su W B, Chang C S and Yao Y D 2007 J. Appl. Phys. 101 09D124

Diaz-Castanon S, Faloh-Gandarilla J C, Munoz-Sandoval E and Terrones M 2008 Superlatt. Microstruct. 43482
Froment B et al 2003 33rd European solid-state device research conference, ESSDERC (Portugal: IEEE Xplore Digital Library) pp 215-218

Joensson C T et al 2006 Nucl. Instrum. Meth. B249 532

Lau S S, Mayer J W and Tu K N 1978 J. Appl. Phys. 494005

Lavoie C, Cabral Jr C, d'Heurle F M, Jordan-Sweet J and Harper J M E 2002 J. Elect. Mater. 31597

Ramaswamy S, Gopalakrishnan C, Kumar N S, Littleflower A and Ponnavaikko M 2010a Appl. Phys. A98 481

Ramaswamy S, Ganesh K R, Gopalakrishnan C and Ponnavaikko M 2010b J. Appl. Phys. 107 09A331

Ramaswamy S, Gopalakrishnan C and Ponnavaikko M 2010c Phys. Scr. 82025603

Ramaswamy S, Gopalakrishnan C, Ganesh K R, Jeganathan K and Ponnavaikko M 2010d J. Vac. Sci. Technol. B28 795

Rao B P C, Ramaswamy S, Gopalakrishnan C, Ganesh K R, Satya Vijayakumar N, John Thiruvadigal D and Ponnavaikko M 2010 Philos. Mag. 903463

Tsay J S, Yao Y D, Wang K C, Cheng W C and Yang C S 2002 J. Appl. Phys. 918766

Wernsdorfer W et al 2009 J. Appl. Phys. 787192 